\title{
Natural fiber reinforced polymer composites in industrial applications: feasibility of date palm fibers for sustainable automotive industry
}

\begin{abstract}
Proper utilization of the available natural resources and wastes became crucial for developing sustainability in industry. In this work, the feasibility of using the date palm fibers in the natural fiber reinforced polymer composites (NFC) for automotive industry was reported. Moreover, this work identifies a gap in the way of evaluating NFC relative to comprehensive desired criteria. This gap leads to disregard potential natural fiber types in industrial applications and keep it no more than an environmental waste problem. Here, criteria that affect the NFC were categorized and classified into levels. Governing criteria were suggested, collected and tabulated according to each level. To ensure the potential and competitiveness of the date palm fiber (DPF) in developing sustainability of the automotive industry, several comparisons between DPF and other fiber types commonly used in this industry were carried out. In most comparisons, DPF was the best selected fiber among all other types. DPF was the best regarding specific Young's modulus to cost ratio criterion. Technical properties and performance, environmental, economical, and societal aspects strongly contribute toward adopting DPF into the automotive sector to improve its sustainability and productivity. Furthermore, this adoption has a significant environmental influence throughout achieving an efficient sustainable waste management practice.
\end{abstract}

Keyword: Industrial sustainability; Natural fibers; Date palm fibers; Waste utilization; Biobased composites; Automotive industry 